BESANÇON

\title{
Calcul des classes de Stiefel-Whitney
} des formes de Pfister 


\title{
Calcul des classes de Stiefel-Whitney des formes de Pfister
}

\author{
Grégory Berhuy
}

Introduction: Le but de ce qui suit est de calculer les classes de StiefelWhitney des formes de Pfister. On en déduira alors celles de la forme trace d'un produit d'algèbres de quaternions, muni d'un produit tensoriel d'involutions de première espèce.

Rappels et notations: Soit $k$ un corps de caractéristique différente de 2. On note $H^{m}(k)$ les groupes de cohomologie $H^{m}\left(G a l\left(k_{\text {sep }} / k\right), \mathbb{Z} / 2 \mathbb{Z}\right)$. Si $a \in k^{*}$, on note $(a)$ l'élément de $H^{1}(k)$ correspondant. Il est bien connu que $H^{1}(k)$ s'identifie à $k^{*} / k^{* 2}$, et que $H^{2}(k)$ s'identifie au noyau de la multiplication par 2 dans le groupe de Brauer de $k$. On rappelle que $(a)+(b)=(a b)$, pour $a, b \in k^{*}$. Si $x \in H^{m}(k)$ et $y \in H^{n}(k)$, on note $x . y$ leur cup-produit. Le cup-produit $(a)$. (b) correspond à l'algèbre de quaternions $(a, b)$ sur $k$. En particulier, on a les relations suivantes, déduites des propriétés des algèbres de quaternions: $(a) \cdot(a)=(a) \cdot(-1),(1) \cdot(a)=0$, et $(a) \cdot(-a)=0$. Etant donné que l'on travaille modulo 2, le cup-produit induit sur $H^{*}(k)=\underset{m \geq 0}{\bigoplus} H^{m}(k)$ une structure d'anneau commutatif. Soit $q$ une forme quadratique non-dégénérée de rang $n$ sur $k$. Si $q \simeq<a_{1}, \cdots, a_{n}>$, et si $r \geq 0$, on définit la r-ième classe de Stiefel-Whitney de $q$ par $w_{r}(q)=\sum_{1 \leq i_{1}<\cdots<i_{r} \leq n}\left(a_{i_{1}}\right) \cdots\left(a_{i_{r}}\right)$. C'est un élément de $H^{r}(k)$, qui ne dépend pas de la diagonalisation de $q$ choisie (cf $[1])$. On a en particulier $w_{0}(q)=1, w_{1}(q)=(\operatorname{det} q)$. Quant à $w_{2}(q)$, c'est l'invariant de Hasse-Witt de $q$. Remarquons que si $m>n$, on a $w_{m}(q)=0$. On définit enfin l'élément de $H^{*}(k)$, appelé classe totale de Stiefel-Whitney de $q, w(q)=\sum_{m \geq 0} w_{m}(q)$. Si $q^{\prime}$ est une autre forme quadratique non-dégénérée sur $k$, on a $w\left(q \perp q^{\prime}\right)=w(q) \cdot w\left(q^{\prime}\right)$ (cf [1]), c'est-à-dire $w_{m}\left(q \perp q^{\prime}\right)=\sum_{j=0}^{m} w_{j}(q) \cdot w_{m-j}\left(q^{\prime}\right)$, pour tout $m \geq 0$.

Si $a_{1}, \cdots, a_{m} \in k^{*},<<a_{1}, \cdots, a_{m}>>$ désigne la forme quadratique

$\bigotimes_{i=1}^{m}<1, a_{i}>$, appelée une $m$-forme de Pfister. Si $m \geq 1$ est un entier, $m q$ 
désigne la somme orthogonale de $m$ copies de $q$.

Si $A$ est une algèbre centrale simple sur $k$, on note $\operatorname{Trd}_{A}$ la trace réduite de $A$. On rappelle qu'une involution $\sigma$ sur $A$ est de première espèce si sa restriction à $k$ est l'identité. La forme $(x, y) \in A \times A \mapsto \operatorname{Trd}_{A}(\sigma(x) y)$ est une forme quadratique sur $k$, que l'on note $\mathcal{T}_{\sigma}$. Pour tout ce qui concerne les algèbres centrales simples, on pourra consulter [4], et plus précisement [2] pour les algèbres à involution.

\section{Effet de la multiplication par un scalaire sur les classes de Stiefel- Whitney}

Lemme 1.1: Soit $A$ un anneau commutatif, et soient $X_{1}, \cdots, X_{n} n$ indéterminées indépendantes. Pour $0 \leq m \leq n$, on note $\sigma_{m}$ le $m$-ième polynôme symétrique élémentaire de $A\left[X_{1}, \cdots, X_{n}\right]$. Alors pour tout $\lambda \in A$, on a $\sigma_{m}\left(X_{1}+\lambda, \cdots, X_{n}+\lambda\right)=\sum_{j=0}^{m} C_{n-m+j}^{j} \lambda^{j} \sigma_{m-j}$.

Preuve: Soit $T$ une autre indéterminée. On a alors $\prod_{l=1}^{n}\left(T+X_{l}\right)=\sum_{j=0}^{n} T^{j} \sigma_{j}$. On a donc $\prod_{l=1}^{n}\left(T+\lambda+X_{l}\right)=\sum_{j=0}^{n} T^{j} \sigma_{j}\left(X_{1}+\lambda, \cdots, X_{n}+\lambda\right)=\sum_{j=0}^{n}(T+\lambda)^{j} \sigma_{j}$ $=\sum_{j=0}^{n} \sum_{i=0}^{j} C_{j}^{i} \lambda^{j-i} T^{i} \sigma_{j}=\sum_{i=0}^{n} T^{i} \sum_{j=i}^{n} C_{j}^{i} \lambda^{j-i} \sigma_{j}=\sum_{i=0}^{n} T^{n-i} \sum_{j=n-i}^{n} C_{j}^{n-i} \lambda^{j-n+i} \sigma_{n-j}$ $=\sum_{i=0}^{n} T^{n-i} \sum_{j=0}^{i} C_{j+n-i}^{n-i} \lambda^{j} \sigma_{j-i}=\sum_{i=0}^{n} T^{n-i} \sum_{j=0}^{i} C_{j+n-i}^{j} \lambda^{j} \sigma_{j-i}$

On a alors le résultat annoncé par identification.

Proposition 1.1: Soit $q$ une forme quadratique non-dégénérée de rang $n$ sur $k$, et soit $\lambda \in k^{*}$. Pour $0 \leq m \leq n$, on a $w_{m}(<\lambda>\otimes q)=$ $\sum_{j=0}^{m} C_{n-m+j}^{j}(\lambda)^{j} \cdot w_{m-j}(q)$.

Preuve: On remarque que si $q \simeq<a_{1}, \cdots, a_{n}>$, on a $w_{m}(q)=\sigma_{m}\left(\left(a_{1}\right), \cdots,\left(a_{n}\right)\right)$, et en tenant compte de l'égalité $\left(\lambda a_{i}\right)=$ $(\lambda)+\left(a_{i}\right)$, il vient $w_{m}(\langle\lambda\rangle \otimes q)=\sigma\left((\lambda)+\left(a_{1}\right), \cdots,(\lambda)+\left(a_{n}\right)\right)$. On obtient alors le résultat en appliquant le lemme 1 avec l'anneau commutatif $A=H^{*}(k)$, et en spécialisant convenablement.

\section{Classes de Stiefel-Whitney des formes de Pfister et produit d'algèbres de quaternions}

On détermine dans ce paragraphe les classes de Stiefel-Whitney des formes de Pfister, ainsi que celles de la forme trace d'un produit d'algèbres de quater- 
nions muni d'un produit tensoriel d'involutions de première espèce.

Si $a_{1}, \cdots, a_{m} \in k^{*}$, on note $q_{m}$ la $m$-forme de Pfister $\left\langle<a_{1}, \cdots, a_{m}>>\right.$.

Proposition 2.1: Si $r \geq 1, r \neq 2^{m-1}$, on a $w_{r}\left(q_{m}\right)=0$.

On aura besoin du lemme suivant:

Lemme 2.1: Si $m \geq 1$, et si $0<r<2^{m}$, on a $C_{2^{m}}^{r} \equiv 0[2]$.

Preuve du lemme 2.1: Il suffit de montrer que $(1+X)^{2^{m}} \equiv 1+X^{2^{m}}$ $\bmod 2 \mathbb{Z}[X]$. Or, ceci est clair pour $m=1$ et le cas général se traite par une récurrence immédiate.

\section{Preuve de la proposition 2.1:}

- On traite d'abord les cas $m=1$ et $m=2$. On a $w_{r}\left(q_{1}\right)=0$ si $r>2$ car $q_{1}$ est de rang 2 , et $w_{2}\left(q_{1}\right)=(1)\left(a_{1}\right)=0$. D'autre part, $w_{1}\left(q_{2}\right)=\left(\operatorname{det} q_{2}\right)=$ $0, w_{3}\left(q_{2}\right)=(1)\left(a_{1}\right)\left(a_{2}\right)+(1)\left(a_{1}\right)\left(a_{1} a_{2}\right)+(1)\left(a_{2}\right)\left(a_{1} a_{2}\right)+\left(a_{1}\right)\left(a_{2}\right)\left(a_{1} a_{2}\right)=$ $\left(a_{1}\right)\left(a_{2}\right)\left(\left(-a_{1}\right)+\left(-a_{2}\right)\right)=\left(a_{1}\right)\left(-a_{1}\right)\left(a_{2}\right)+\left(a_{1}\right)\left(a_{2}\right)\left(-a_{2}\right)=0$, et $w_{4}\left(q_{2}\right)=$ (1) $\left(a_{1}\right)\left(a_{2}\right)\left(a_{1} a_{2}\right)=0$. Le résultat est donc vrai si $m=1$ et $m=2$.

- On procède par récurrence sur $m$. D'après le point précédent, la proposition est vraie pour $m=2$. Supposons qu'elle est vraie pour $m \geq 2$. On a $q_{m+1}=$ $q_{m} \perp<a_{m+1}>\otimes q_{m}$, et donc $w_{r}\left(q_{m+1}\right)=\sum_{j=0}^{r} w_{j}\left(q_{m}\right) w_{r-j}\left(<a_{m+1}>\otimes q_{m}\right)$. On traite plusieurs cas séparément.

1. $1 \leq r<2^{m-1}$

L'hypothèse de récurrence entraîne alors $w_{r}\left(q_{m+1}\right)=w_{r}\left(<a_{m+1}>\otimes q_{m}\right)$ $=\sum_{j=0}^{r} C_{2^{m}-r+j}^{j}\left(a_{m+1}\right)^{j} \cdot w_{r-j}\left(q_{m}\right)=C_{2^{m}}^{r}\left(a_{m+1}\right)^{r}=0$, d'après le lemme 3.1 .

2. $r=2^{m-1}$

On a dans ce cas $w_{2^{m-1}}\left(q_{m+1}\right)=w_{2^{m-1}}\left(q_{m}\right)+w_{2^{m-1}}\left(<a_{m+1} \otimes q_{m}>\right.$ )$=w_{2^{m-1}}\left(q_{m}\right)+\sum_{j=0}^{2^{m-1}} C_{2^{m-2^{m-1}+j}}^{j}\left(a_{m+1}\right)^{j} w_{2^{m-1}-j}\left(q_{m}\right)=w_{2^{m-1}}\left(q_{m}\right)+$ $w_{2^{m-1}}\left(q_{m}\right)+C_{2^{m}}^{2^{m-1}}\left(a_{m+1}\right)^{2^{m-1}}=0$, car on travaille modulo 2 .

3. $2^{m-1}<r<2^{m}$

Ici on a $w_{r}\left(q_{m+1}\right)=w_{r}\left(q_{m}\right)+w_{r}\left(<a_{m+1}>\otimes q_{m}\right)+w_{2^{m-1}}\left(q_{m}\right) \cdot w_{r-2^{m-1}}(<$ 
$\left.a_{m+1}>\otimes q_{m}\right)$. Par hypothèse, on a $w_{r}\left(q_{m}\right)=0$. D'autre part, $0<r-$ $2^{m-1}<2^{m-1}$. D'après le calcul effectué dans le premier cas, on a alors $w_{r-2^{m-1}}\left(<a_{m+1} \otimes q_{m}\right)=0$. De plus, $w_{r}\left(<a_{m+1} \otimes q_{m}\right)=C_{2^{m}}^{r}\left(a_{m+1}\right)^{r}+$ $C_{2^{m-1}}^{r-2^{m-1}}\left(a_{m+1}\right)^{r-2^{m-1}} \cdot w_{2^{m-1}}\left(q_{m}\right)=0$ d'après le lemme 2. On a donc $w_{r}\left(q_{m+1}\right)=0$.

4. $2^{m}<r<2^{m+1}$ On a $w_{r}\left(q_{m+1}\right)=w_{r}\left(q_{m}\right)+w_{r}\left(<a_{m+1}>\otimes q_{m}\right)+$ $w_{2^{m-1}}\left(q_{m}\right) \cdot w_{r-2^{m-1}}\left(<a_{m+1}>\otimes q_{m}\right)$. Par hypoyhèse, on a $w_{r}\left(q_{m}\right)=0$, et on montre comme précédemment que $w_{r}\left(\left\langle a_{m+1} \otimes q_{m}\right\rangle\right)=0$. Enfin, $w_{r-2^{m-1}}\left(<a_{m+1}>\otimes q_{m}\right)=\sum_{j=0}^{r-2^{m-1}} C_{2^{m}-\left(r-2^{m-1}\right)+j}^{j}\left(a_{m+1}\right)^{j} w_{r-2^{m-1}-j}\left(q_{m}\right)=$ $C_{2^{m}}^{r}\left(a_{m+1}\right)^{r-2^{m-1}}+C_{2^{m-1}}^{r-2^{m}} w_{2^{m-1}}\left(q_{m}\right)=0$, et donc $w_{r}\left(q_{m+1}\right)=0$.

Finalement, la proposition est vraie au rang $m+1$, ce qui achève la récurrence.

Proposition 2.2: Pour $m \geq 1$, on a $w_{2^{m-1}}\left(q_{m}\right)=\sum_{j=1}^{m}\left(a_{j}\right)\left(-a_{j+1}\right)\left(-a_{j+2}\right) \cdots\left(-a_{m}\right)(-1)^{2^{m-1}-m+j-1}$.

Preuve: On a $w_{1}\left(q_{1}\right)=\left(a_{1}\right)$ et $w_{2^{m}}\left(q_{m+1}\right)=\sum_{j=0}^{2^{m}} w_{j}\left(q_{m}\right) w_{2^{m}-j}\left(<a_{m+1}>\otimes q_{m}\right)$ $=w_{2^{m-1}}\left(q_{m}\right) w_{2^{m-1}}\left(<a_{m+1}>\otimes q_{m}\right)+w_{2^{m}}\left(<a_{m+1}>\otimes q_{m}\right)$ grâce à la proposition précédente. On obtient par le même argument $w_{2^{m}}\left(<a_{m+1}>\otimes q_{m}\right)=$ $\left(a_{m+1}\right)^{2^{m}}+w_{2^{m-1}}\left(q_{m}\right) \cdot\left(a_{m+1}\right)^{2^{m-1}}$ et $w_{2^{m-1}}\left(<a_{m+1}>\otimes q_{m}\right)=w_{2^{m-1}}\left(q_{m}\right)$. On a donc $w_{2^{m}}\left(q_{m+1}\right) \doteq w_{2^{m-1}}\left(q_{m}\right)^{2}+\left(a_{m+1}\right)^{2^{m}}+\left(a_{m+1}\right)^{2^{m-1}}\left(q_{m}\right)$ pour tout $m \geq 1$. Une récurrence immédiate nous montre que $(\lambda)^{2^{m}}=(\lambda)(-1)^{2^{m}-1}$ pour $\lambda \in k^{*}$. D'autre part, on voit facilement, en tenant compte que l'on travaille modulo 2 et en utilisant le fait que $(a)(a)=(-1)(a)$, que $w_{2^{m-1}}\left(q_{m}\right)^{2}=(-1)^{2^{m-1}} \cdot w_{2^{m-1}}\left(q_{m}\right)$.

On a donc $w_{2^{m}}\left(q_{m+1}\right)=\left[(-1)^{2^{m-1}-1} \cdot\left(a_{m+1}\right)+(-1)^{2^{m-1}}\right] \cdot w_{2^{m-1}}\left(q_{m}\right)$ $+(-1)^{2^{m}-1}\left(a_{m+1}\right)=(-1)^{2^{m-1}-1} \cdot\left[\left(a_{m+1}\right)+(-1)\right] \cdot w_{2^{m-1}}\left(q_{m}\right)+(-1)^{2^{m}-1}\left(a_{m+1}\right)$. On a alors $w_{2^{m}}\left(q_{m+1}\right)=(-1)^{2^{m-1}-1}\left(-a_{m+1}\right) \cdot w_{2^{m-1}}\left(q_{m}\right)+(-1)^{2^{m}-1}\left(a_{m+1}\right)$. On en déduit alors la proposition par une récurrence immédiate.

On suppose maintenant que $A$ est un produit d'algèbres de quaternions. $A$ est donc en particulier une algèbre à involution. On a alors le résultat suivant:

Théorème 2.1: Soient $Q_{1}, \cdots, Q_{m}$ des algèbres de quaternions, munies respectivement d'involutions de première espèce $\sigma_{1}, \cdots, \sigma_{m}$. On pose $A=$ $Q_{1} \otimes \cdots \otimes Q_{m}$ et $\sigma=\sigma_{1} \otimes \cdots \sigma_{m}$. Alors il existe $a_{1}, \cdots, a_{2 m} \in k^{*}$ tels que $T_{\sigma} \simeq<2^{m}>\otimes<a_{1}, \cdots, a_{2 m}>>$. En particulier, $w\left(T_{\sigma}\right)=1+\sum_{j=1}^{2 m}\left(a_{j}\right)\left(-a_{j+1}\right)\left(-a_{j+2}\right) \cdots\left(-a_{2 m}\right)(-1)^{2^{2 m-1}-2 m+j-1}$. 
Preuve: Il est bien connu que si $\left(A_{1}, \sigma_{1}\right)$ et $\left(A_{2}, \sigma_{2}\right)$ sont deux algèbres centrales simples sur $k$, dont les involutions sont de même espèce, alors $\mathcal{T}_{\sigma_{1} \otimes \sigma_{2}} \simeq \mathcal{T}_{\sigma_{1}} \otimes \mathcal{T}_{\sigma_{2}}$. Si $\sigma_{i}$ est symplectique, on sait alors que c'est l'involution canonique de $Q_{i}$. Si on a $Q_{i}=(\alpha, \beta)$, alors on en déduit aisément $\mathcal{T}_{\sigma_{i}}$ $\simeq<2>\otimes<<-\alpha,-\beta>>$. Si $\sigma_{i}$ est orthogonale, on sait alors qu'il existe deux éléments $i, j$ de $Q_{i}$ tels que $1, i, j, i j$ soit une base de $Q_{i}$ sur $k$, orthogonale pour $\mathcal{T}_{\sigma_{i}}$. Posons $\alpha=i^{2}$ et $\beta=j^{2}$. Alors $Q_{i}=(\alpha, \beta)$ et $\mathcal{T}_{\sigma_{i}}$ $\simeq<2>\otimes<<-\alpha, \beta>>$ (cf.[3], §2.1.2). Enfin, un calcul facile montre que $w_{2^{2 m-1}}(<2>\otimes q)=w_{2^{2 m-1}}(q)$, si $q$ est une $2 m$-forme de Pfister. Les propositions 2.1 et 2.2 nous donnent alors le résultat.

\section{Bibliographie}

[1] Delzant A. Définition des classes de Stiefel-Whitney d'un module quadratique sur un corps de caractéristique différente de 2. C.R.Acad.Sci. Paris 255, 1366-1368 (1962)

[2] Knus M.-A., Merkurjev A., Rost M., Tignol J.-P. The Book of Involutions. A.M.S. Colloquium Publications 44 (1998)

[3] Queguiner A. Invariants d'algèbres à involution. Thèse de l'université de Besançon $n^{\circ} 555$ (1996)

[4] Scharlau W. Quadratic and Hermitian Forms. Grundleheren Math. Wiss. vol. 270. Springer-Verlag. New York (1985)

Adresse:

Equipe de Mathématiques de Besançon

UMR 6623 du C.N.R.S.

16 , route de Gray

25030 Besançon Cedex

France 